\title{
The Intriguing Distribution of Dark Matter in Galaxies
}

\author{
Paolo Salucci ${ }^{1}$ and Annamaria Borriello ${ }^{1}$ \\ (1) International School for Advanced Studies SISSA-ISAS - Trieste, I
}

\begin{abstract}
We review the most recent evidence for the amazing properties of the density distribution of the dark matter around spiral galaxies. Their rotation curves, coadded according to the galaxy luminosity, conform to an Universal profile which can be represented as the sum of an exponential thin disk term plus a spherical halo term with a flat density core. From dwarfs to giants, these halos feature a constant density region of size $r_{0}$ and core density $\rho_{0}$ related by $\rho_{0}=4.5 \times 10^{-2}\left(r_{0} / \mathrm{kpc}\right)^{-2 / 3} \mathrm{M}_{\odot} \mathrm{pc}^{-3}$. At the highest masses $\rho_{0}$ decreases exponentially with $r_{0}$, revealing a lack of objects with disk masses $>10^{11} M_{\odot}$ and central densities $>1.5 \times 10^{-2}\left(r_{0} / \mathrm{kpc}\right)^{-3} M_{\odot} \mathrm{pc}^{-3}$ implying a maximum mass of $\approx 2 \times 10^{12} M_{\odot}$ for a dark halo hosting a stellar disk. The fine structure of dark matter halos is obtained from the kinematics of a number of suitable low-luminosity disk galaxies. The halo circular velocity increases linearly with radius out to the edge of the stellar disk, implying a constant dark halo density over the entire disk region. The properties of halos around normal spirals provide substantial evidence of a discrepancy between the mass distributions predicted in the Cold Dark Matter scenario and those actually detected around galaxies.
\end{abstract}

\section{Introduction}

Rotation curves (RC's) of disk galaxies are the best probe for dark matter (DM) on galactic scale. Notwithstanding the impressive amount of knowledge gathered in the past 20 years, only very recently we start to shed light to crucial aspects of the mass distribution of dark halos, including their radial density profile, and its claimed universality. On a cosmological side, high-resolution N-body simulations have shown that cold dark matter $(\mathrm{CDM})$ halos achieve a specific equilibrium density profile [13 hereafter NFW, 5, 8, 12, 9] characterized by one free parameter, e.g. the halo mass. In the inner region the DM halos density profiles show some scatter around an average profile which is characterized by a power-law cusp $\rho \sim r^{-\gamma}$, with $\gamma=1-1.5[13,12,2]$. In detail, the DM density profile is:

$$
\rho_{\mathrm{NFW}}(r)=\frac{\rho_{s}}{\left(r / r_{s}\right)\left(1+r / r_{s}\right)^{2}}
$$

where $r_{s}$ and $\rho_{s}$ are respectively the characteristic inner radius and density. Let us define $r_{\text {vir }}$ as the radius within which the mean density is $\Delta_{\text {vir }}$ times the mean universal density $\rho_{m}$ at the halo formation redshift, and the associated virial mass $M_{\text {vir }}$ and velocity $V_{\text {vir }} \equiv G M_{\text {vir }} / r_{\text {vir }}$. Hereafter we assume the $\Lambda \mathrm{CDM}$ scenario, with $\Omega_{m}=0.3, \Omega_{\Lambda}=0.7$ and $h=0.75$, so that $\Delta_{\text {vir }} \simeq 340$ at $z \simeq 0$. By 

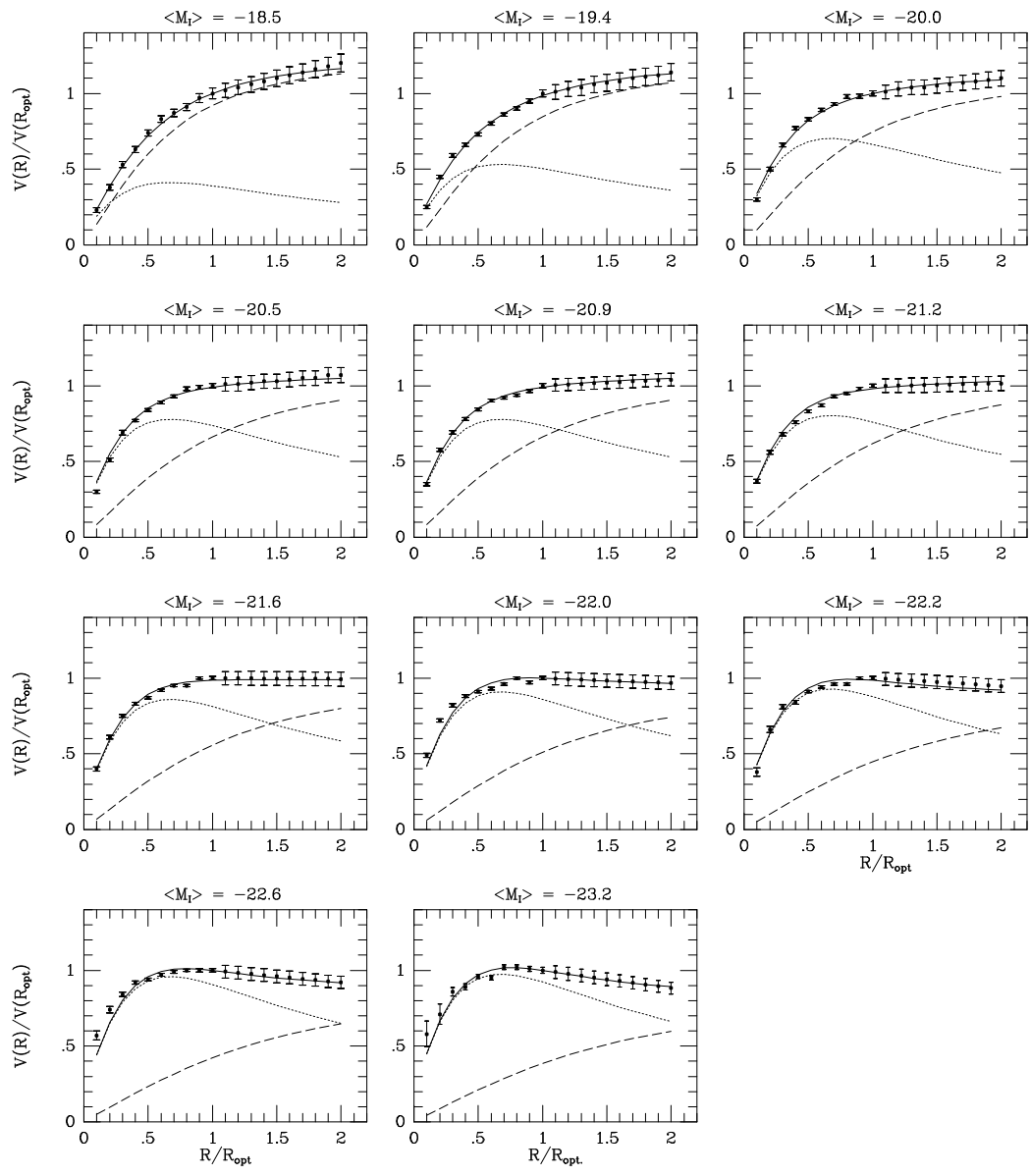

Fig. 1. Synthetic rotation curves (filled circles with error bars) and URC (solid line) with the separate dark/luminous contributions (dotted line: disks; dashed line: halos).

assuming the concentration parameter as $c \equiv r_{\mathrm{vir}} / r_{s}$ the halo circular velocity $V_{\mathrm{NFW}}(r)$ takes the form [2]:

$$
V_{\mathrm{NFW}}^{2}(r)=V_{v i r}^{2} \frac{c}{A(c)} \frac{A(x)}{x}
$$

where $x \equiv r / r_{s}$ and $A(x) \equiv \ln (1+x)-x /(1+x)$. As the relation between $V_{v i r}$ and $r_{v i r}$ is fully specified by the background cosmology, the independent parameters characterizing the model reduce from three to two $\left(c\right.$ and $\left.r_{s}\right)$. Let us stress that a high density $\Omega_{m}=1$ model, with a concentration parameter $c>12$, is definitely unable to account for the observed galaxy kinematics [11].

So far, due to the limited number of suitable RC's and to the serious uncertainties in deriving the actual amount of luminous matter inside the inner 
regions of spirals, it has been difficult to investigate the internal structure of dark halos. These difficulties have been overcome by means of:

i) a specific investigation of the Universal Rotation Curve [16], built by coadding 1000 RC's, in which we adopt a general halo mass distribution:

$$
V_{h, U R C}^{2}(x)=V_{o p t}^{2}(1-\beta)\left(1+a^{2}\right) \frac{x^{2}}{\left(x^{2}+a^{2}\right)}
$$

with $x \equiv r / r_{o p t}$, $a$ the halo core radius in units of $r_{\text {opt }}$ and $\beta \equiv\left(V_{d, U R C}\left(r_{o p t}\right) / V_{o p t}\right)^{2}$. It is important to remark that, out to $r_{\text {opt }}$, this mass model is neutral with respect to the halo profile. Indeed, by varying $\beta$ and $a$, we can efficiently reproduce the maximum-disk, the solid-body, the no-halo, the all-halo, the CDM and the core-less-halo models. For instance, CDM halos with concentration parameter $c=5$ and $r_{s}=r_{\text {opt }}$ are well fit by (3) with $a \simeq 0.33$

ii) a number of suitably selected individual RC's [1], whose mass decomposition has been made adopting the cored Burkert-Borriello-Salucci (BBS) halo profile (see below).

\section{Dark Matter Properties from the Universal Rotation Curve}

The observational framework is the following: $a$ ) the mass in spirals is distributed according to the Inner Baryon Dominance (IBD) regime [16]: there is a characteristic transition radius $r_{I B D} \simeq 2 r_{d}\left(V_{\text {opt }} / 220 \mathrm{~km} / \mathrm{s}\right)^{1.2}\left(r_{d}\right.$ is the disk scale-length and $\left.V_{o p t} \equiv V\left(r_{o p t}\right)\right)$ according which, for $r \leq r_{I B D}$, the luminous matter totally accounts for the gravitating mass, whereas, for $r>r_{I B D}$, the dark matter shows dynamically up and rapidly becomes the dominant component $[20,18,1]$. Then, although dark halo might extend down to the galaxy centers, it is only for $r>r_{I B D}$ that they give a non-negligible contribution to the circular velocity. $b$ ) DM is distributed in a different way with respect to any of the various baryonic components $[16,6]$, and $c$ ) HI contribution to the circular velocity at $r<r_{\text {opt }}$, is negligible [e.g. 17].

\subsection{Mass modeling}

Persic, Salucci and Stel [16] have derived from $\sim 15000$ velocity measurements of $\sim 1000$ RC's the synthetic rotation velocities of spirals $V_{\text {syn }}\left(\frac{r}{r_{\text {opt }}} ; \frac{L_{I}}{L_{*}}\right)$, sorted by luminosity (Fig. 1, with $L_{I}$ the $I$-band luminosity $\left(L_{I} / L_{*}=10^{-\left(M_{I}+21.9\right) / 5}\right)$. Remarkably, individual RC's have a negligible variance with respect to their corresponding synthetic curves: spirals sweep a very narrow locus in the RCprofile/amplitude/luminosity space. In addition, kinematical properties of spirals do significantly change with galaxy luminosity [e.g. 16], then it is natural to relate their mass distribution with this quantity. The whole set of synthetic RC's define the Universal Rotation Curve (URC), composed by the sum of two terms: $a$ ) an exponential thin disk with circular velocity (see [16]):

$$
V_{d, U R C}^{2}(x)=\left.1.28 \beta V_{o p t}^{2} x^{2}\left(I_{0} K_{0}-I_{1} K_{1}\right)\right|_{1.6 x}
$$



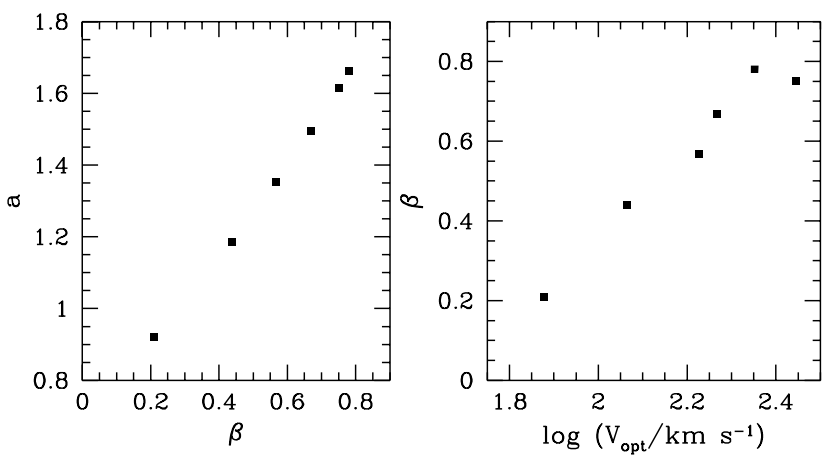

Fig. 2. $a$ vs. $\beta$ and $\beta$ vs. $V_{o p t}$.

and a spherical halo, whose velocity contribution is given by (3). At high luminosities, the contribution from a bulge component has also been considered.

The data (i.e. the synthetic curves $V_{\text {syn }}$ ) select the actual model out of this family, by setting $V_{U R C}^{2}(x)=V_{h, U R C}^{2}(x, \beta, a)+V_{d, U R C}^{2}(x, \beta)$ with $a$ and $\beta$ as free parameters. An extremely good fit occurs for $a \simeq 1.5\left(L_{I} / L_{*}\right)$ [16] or, equivalently, for $a=a(\beta)$ and $\beta=\beta\left(\log V_{o p t}\right)$ as plotted in Fig. 2. With these values the URC reproduces the data $V_{\text {syn }}(r)$ up to their $r m s$ (i.e. within $2 \%$ ). Moreover, at fixed luminosity the $\sigma$ fitting uncertainties in $a$ and $\beta$ are lesser than $20 \%$. The emerging picture is: $i$ ) smaller objects have more fractional amount of dark matter $\left(\right.$ inside $\left.r_{\mathrm{opt}}: M_{*} / M_{\mathrm{vir}} \simeq 0.2\left(M_{*} / 2 \times 10^{11} M_{\odot}\right)^{0.75}[20]\right)$, ii) dark mass increses with radius much more that linearly.

\subsection{Halo Density Profiles}

The above evidence calls for a quite specific DM density profile; we adopt the BBS halo mass distribution $[3,4,1]$ :

$$
\rho_{\mathrm{BBS}}(r)=\frac{\rho_{0} r_{0}^{3}}{\left(r+r_{0}\right)\left(r^{2}+r_{0}^{2}\right)}
$$

where $\rho_{0}$ and $r_{0}$ are free parameters which represent the central DM density and the core radius. Of course, for $r_{0} \ll r_{d}$, we recover a cuspy profile. Within spherical symmetry, the mass distribution is given by:

$$
\begin{gathered}
M_{\mathrm{BBS}}(r)=4 M_{0}\left\{\ln \left(1+r / r_{0}\right)-\arctan \left(r / r_{0}\right)+0.5 \ln \left[1+\left(r / r_{0}\right)^{2}\right]\right\} \\
M_{0} \simeq 1.6 \rho_{0} r_{0}^{3}
\end{gathered}
$$

with $M_{0}$ the dark mass within the core. The halo contribution to the circular velocity is then: $V_{\mathrm{BBS}}^{2}(r)=G M_{\mathrm{BBS}}(r) / r$. Although the dark matter "core" parameters $r_{0}, \rho_{0}$ and $M_{0}$ are in principle independent, the observations reveal a quite strong correlation among them [e.g. 19]. Then, dark halos may be an 

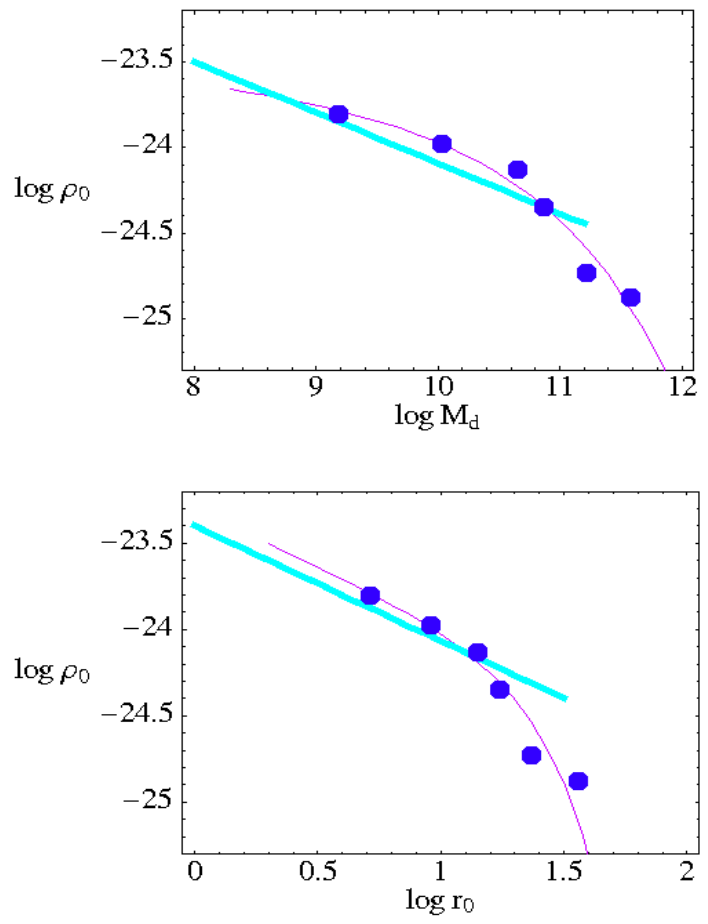

Fig. 3. up) Central halo density $\rho_{0}\left(\mathrm{in} \mathrm{g} / \mathrm{cm}^{3}\right)$ vs. disk mass (in solar units) for normal spirals (filled circles); bottom) central density vs. core radii (in $\mathrm{kpc}$ ) for normal spirals. The straight lines are from [3], whereas the curved lines are the best fits used in $\S 4$.

1-parameter family, completely specified by e.g. their core mass $M_{0}$. When we test the disk+BBS velocities with $\rho_{0}$ and $r_{0}$ left as free parameters, we find that, at any luminosity and out to $\sim 6 r_{d}$, the model is indistinguishable from data (i.e. $V_{\text {syn }}(r)$ ). More specifically, we reproduce the synthetic rotation curves at the level of their rms. The values of $r_{0}$ and $\rho_{0}$ derived in this way agree with the extrapolation at high masses of the scaling law $\rho \propto r_{0}^{-2 / 3}[3]$ established for objects with much smaller core radii $r_{0}$ and stellar masses (see Fig. 3). Let us notice that the core radii are pretty large $\left(r_{0} \gg r_{d}\right)$ : ever-rising halo RC's cannot be excluded by the data. Moreover, spirals lie on the extrapolation of the disk-mass vs. central halo density relationship $\rho_{0} \propto M_{d}^{-1 / 3}$ found for dwarf galaxies [3], to indicate that the densest halos harbor the least massive disks (see Fig. 3).

The curvature in $\rho_{0}$ vs. $r_{0}$ at the highest masses/lowest densities can be linked to the existence of an upper mass limit in $M_{\text {vir }}$ which is evident by the sudden decline of the baryonic mass function of disk galaxies at $M_{d}^{\max }=2 \times 10^{11} M_{\odot}[20]$. 
In fact, such a limit implies a maximum halo mass of $M_{\text {vir }}^{\max } \sim \Omega_{0} / \Omega_{b} M_{d}^{\max }$. Then, for (6) and (7), $M_{\text {vir }}=\eta M_{0}$, with $\eta \simeq 12$ for $\left(\Omega_{0}, \Omega_{b}, z\right)=(0.3,0.03,3)$, and the limiting halo mass implies a lack of objects with $\rho_{0}>4 \times 10^{-25} \mathrm{~g} / \mathrm{cm}^{3}$ and $r_{0}>30 \mathrm{kpc}$, as is evident in Fig. 3. On the other side, the observed deficit of objects with $M_{d} \sim M_{d}^{\max }$ and $\rho_{0}>4 \times 10^{-25} \mathrm{~g} / \mathrm{cm}^{3}$, suggests that, at this mass scale, the total-to-baryonic density ratio nears the cosmological value $\Omega_{0} / \Omega_{b} \simeq 10$

\subsection{Testing CDM with the URC}

The BBS density profile reproduces in synthetic RC's the DM halo contributions, at least out to two optical radii. This is in contradiction with CDM halo properties according to which the velocity dispersion $\sigma$ of the dark matter particles decreases towards the center to reach $\sigma \rightarrow 0$ for $r \rightarrow 0$. Dark halos therefore, are not kinematically cold structures, but "warm" regions of sizes $r_{0} \propto \rho_{0}^{-1.5}$ which, by the way, turn up quite large: $r_{0} \sim 4-7 r_{d}$. Then, the boundary of the core region is well beyond the region of the stellar disk and there is not evidence that dark halos converge to a $\rho \sim r^{-2}$ (or a steeper) regime, as dictated by CDM predictions.

\section{Dark Matter Properties from Individual Rotation Curves}

Although deriving halo densities from individual RC's is certainly complicated, the belief according to which one always gets ambiguous halo mass modeling [e.g. 22] is incorrect. In fact, this is true only for rotation curves of low spatial resolution, i.e. with less than $\sim 3$ measures per exponential disk length-scale occurring in most HI RC's. In this case, since the parameters of the galaxy structure are very sensitive to the shape of the rotation curve in the region $0<r<r_{d}$, there are no sufficient data to constrain models.

In the case of high-quality optical RC's tens of independent measurements in the critical region make possible to infer the halo mass distribution. Moreover, since the dark component can be better traced when the disk contributes to the dynamics in a modest way, a convenient strategy leads to investigate DMdominated objects, like dwarf and low surface brightness (LSB) galaxies. It is well known that for the latter [e.g. 7, 11, 3, 4, 9, 10, 21] the results are far from being definitive in that they are 1) affected by a quite low spatial resolution and 2) uncertain, due to the limited amount of available kinematical data [e.g. 23].

Since most of the properties of cosmological halos are claimed universal, an useful strategy is to investigate a number of high-quality optical rotation curves of low luminosity late-type spirals, with $I$-band absolute magnitudes $-21.4<M_{I}<-20.0$ and $100<V_{\text {opt }}<170 \mathrm{~km} \mathrm{~s}^{-1}$. Objects in this luminosity/velocity range are DM dominated [e.g. 20] but their RC's, measured at an angular resolution of $2^{\prime \prime}$, have an excellent spatial resolution of $\sim 100(D / 10 \mathrm{Mpc})$ pc and $n_{\text {data }} \sim r_{o p t} / w$ independent measurements. For nearby galaxies: $w<<r_{d}$ 


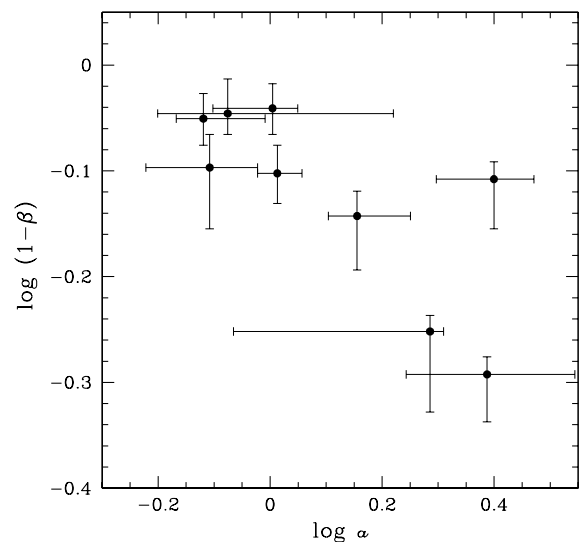

Fig. 4. Halo parameters ( $a$ is in units of $r_{\text {opt }}$ ) for the individual RC's. Notice that for this sample we can derive in model-independent way $a \gtrsim 1$, in disagreement with CDM predictions.

and $n_{\text {data }}>25$. Moreover, we select RC's of bulge-less systems, so that the stellar disk is the only baryonic component for $r \lesssim r_{d}$.

In detail, we extract the best 9 rotation curves, from the 'excellent' subsample of 80 rotation curves of [15], which are all suitable for an accurate mass modeling. In fact, these RC's trace properly the gravitational potential in that: 1) data extend at least out to the optical radius, 2) they are smooth and symmetric, 3) they have small rms, 4) they have high spatial resolution and a homogeneous radial data coverage, i.e. about $30-100$ data points homogeneously distributed with radius and between the two arms. The 9 extracted galaxies are of low luminosity $\left(5 \times 10^{9} L_{\odot}<L_{I}<2 \times 10^{10} L_{\odot} ; 100<V_{o p t}<170 \mathrm{~km} \mathrm{~s}^{-1}\right)$ and their $I$-band surface luminosity profiles are (almost) perfect radial exponential. These two last criteria, not indispensable to perform the mass decomposition, help inferring the dark halo density distribution. Each RC has $7-15$ velocity points inside $r_{\text {opt }}$, each one being the average of $2-6$ independent data. The RC's spatial resolution is better than $1 / 20 r_{o p t}$, the velocity $r m s$ is about $3 \%$ and the RC's logarithmic derivative is generally known within about 0.05.

\subsection{Halo Density Profiles}

We model the mass distribution as the sum of two components: a stellar disk and a spherical dark halo. By assuming centrifugal equilibrium under the action of the gravitational potential, the observed circular velocity can be split into the two components: $V^{2}(r)=V_{d}^{2}(r)+V_{h}^{2}(r)$. By selection, the objects are bulge-less and stars are distributed like an exponential thin disk. Light traces the mass via an assumed radially constant mass-to-light ratio. 

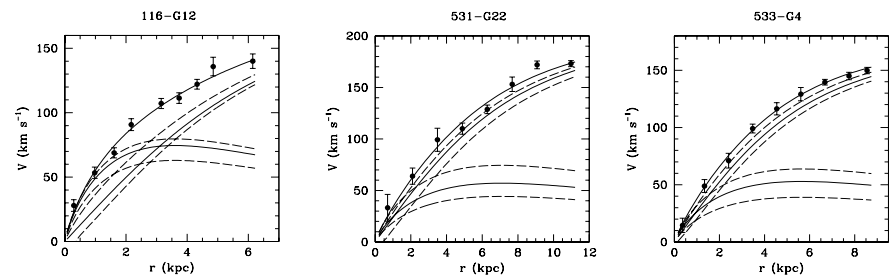

545- 65

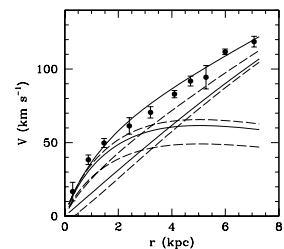

$563-\mathrm{G} 14$
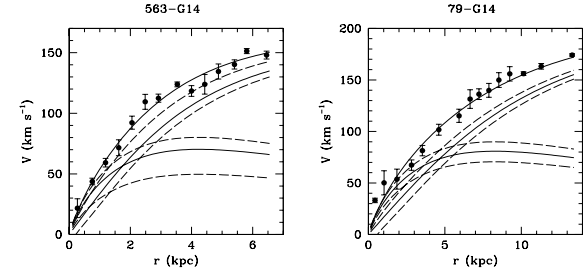

$\mathrm{M}-3-104$

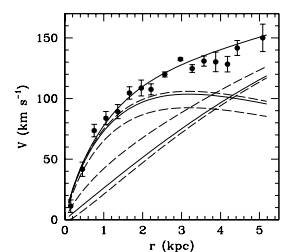

N733
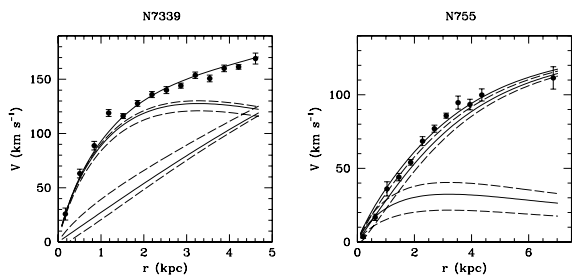

Fig. 5. BBS fits (thick solid line) to the RC's (points with errorbars). Thin solid lines represent the disk and halo contributions. Notice the steep halo velocity profiles. The maximum and minimum disk solutions (dashed lines) provide the theoretical uncertainties.

We neglect the gas contribution $V_{\text {gas }}(r)$ since in normal spirals it is usually modest within the optical region [17, Fig. 4.13]: $\beta_{\text {gas }} \equiv\left(V_{\text {gas }}^{2} / V^{2}\right)_{r_{\text {opt }}} \sim 0.1$. Furthermore, high resolution HI observations show that in low luminosity spirals: $V_{\text {gas }}(r) \simeq 0$ for $r<r_{d}$ and $V_{\text {gas }}(r) \simeq(20 \pm 5)\left(r-r_{d}\right) / 2 r_{d}$ for $r_{d} \leq r \leq 3 r_{d}$. Thus, in the optical region: i) $V_{\text {gas }}^{2}(r)<<V^{2}(r)$ and ii) $d\left[V^{2}(r)-V_{\text {gas }}^{2}(r)\right] / d r \gtrsim 0$. This last condition implies that by including $V_{\text {gas }}$ the halo velocity profiles would result steeper and then the core radius in the halo density even larger. Incidentally, this is not the case for dwarfs and LSBs: most of their kinematics is affected by the HI disk gravitational pull in such a way that neglecting it could bias the determination of the DM density. The circular velocity profile of the disk is given by (4) and the DM halo will have the form given by (3). Since we normalize (at $r_{\text {opt }}$ ) the velocity model $\left(V_{d}^{2}+V_{h}^{2}\right)^{1 / 2}$ to the observed rotation speed $V_{\text {opt }}, \beta$ enters explicitly in the halo velocity model and this reduces the free parameters of the mass model to two.

For each galaxy, we determine the values of the parameters $\beta$ and $a$ by means of a $\chi^{2}$-minimization fit to the observed rotation curves:

$$
V_{\text {model }}^{2}(r ; \beta, a)=V_{d}^{2}(r ; \beta)+V_{h}^{2}(r ; \beta, a)
$$



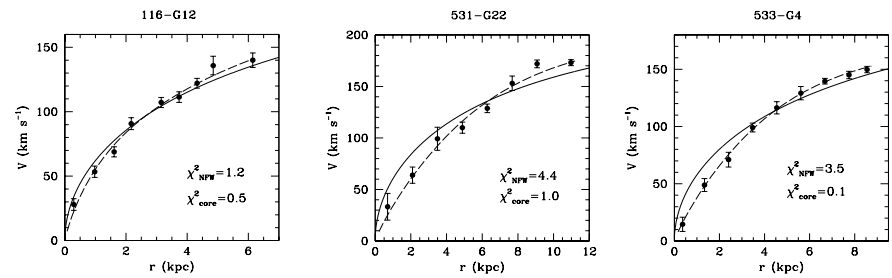

$545-65$

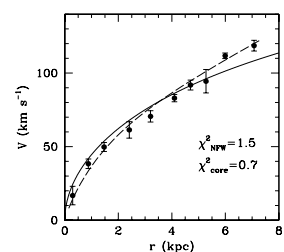

$563-\mathrm{G} 14$
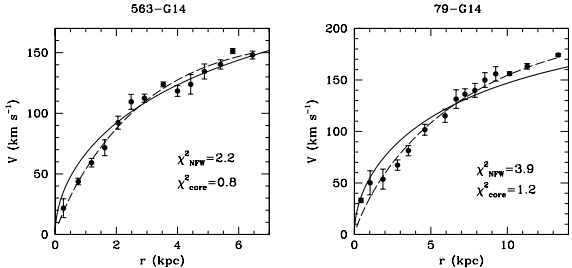

$x-3-1040$
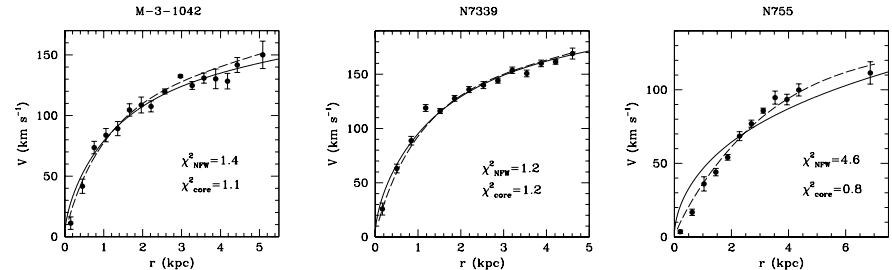

Fig. 6. NFW best-fits solid lines of the rotation curves (filled circles) compared with the BBS fits (dashed lines). The $\chi^{2}$ values are also indicated.

A central role in discriminating among the different mass decompositions is played by the derivative of the velocity field $d V / d r$. It has been shown [e.g. 14] that by taking into account the logarithmic gradient of the circular velocity field defined as: $\nabla(r) \equiv \frac{d \log V(r)}{d \log r}$ one can retrieve the crucial information stored in the shape of the rotation curve. Then, we set the $\chi^{2}$ s as the sum of those evaluated on velocities and on logarithmic gradients: $\chi_{V}^{2}=\sum_{i=1}^{n_{V}} \frac{V_{i}-V_{\text {model }}\left(r_{i} ; \beta, a\right)}{\delta V_{i}}$ and $\chi_{\nabla}^{2}=\sum_{i=1}^{n_{\nabla}} \frac{\nabla\left(r_{i}\right)-\nabla_{\text {model }}\left(r_{i} ; \beta, a\right)}{\delta \nabla_{i}}$, with $\nabla_{\text {model }}\left(r_{i}, \beta, a\right)$ given from the above equations. The parameters of the mass models are finally obtained by minimizing the quantity $\chi_{\text {tot }}^{2} \equiv \chi_{V}^{2}+\chi_{\nabla}^{2}$.

The best-fit models parameters are shown in Fig. 4. The disk-contribution $\beta$ and the halo core radius $a$ span a range from 0.1 to 0.5 and from 0.8 to 2.5 , respectively. They are pretty well constrained in a small and continuous region of the $(a, \beta)$ space. The derived mass models are shown in Fig. 5 , alongside with the separate disk and halo contributions. We also get a "lowest" and a "highest" halo velocity curve (dashed lines in figure) by subtracting from $V(r)$ the maximum and the minimum disk contributions $V_{d}(r)$ obtained by substituting in (4) the parameter $\beta$ with $\beta_{\text {best }}+\delta \beta$ and $\beta_{\text {best }}-\delta \beta$, respectively. It is obvious that the halo curves are steadily increasing, almost linearly, out to the last data point. In each object the uniqueness of the resulting halo velocity model can be realized 
by the fact that the maximum-disk and minimum-disk models almost coincide. Remarkably, we find that the size of the halo density core is always greater than the disk characteristic scale-length $r_{d}$ and it can extend beyond the disk edge (and the region investigated).

\subsection{Testing CDM}

In Fig. 5 we show how the halo velocity profiles of the nine galaxies rise almost linearly with radius, at least out to the disk edge:

$$
V_{h}(r) \propto r \quad 0.05 r_{\text {opt }} \lesssim r \lesssim r_{\text {opt }}
$$

The halo density profile has a well defined (core) radius within which the density is approximately constant. This is inconsistent with the singular halo density distribution emerging in the Cold Dark Matter (CDM) halo formation scenario. More precisely, since the CDM halos are, at small radii, likely more cuspy than the NFW profile: $\rho_{C D M} \propto r^{-1.5}$ [e.g. 14], the steepest CDM halo velocity profile $V_{h}(r) \propto r^{1 / 4}$ results too shallow with respect to observations. Although the mass models of (3) converge to a distribution with an inner core rather than with a central spike, it is worth, given the importance of such result, checking in a direct way the (in)compatibility of the CDM models with galaxy kinematics.

So, we assume the NFW functional form for the halo density given by (1), leaving $c$ and $r_{s}$ as free independent parameters, although $\mathrm{N}$-body simulations and semi-analytic investigations indicate that they correlate. This choice to increase the chance of a good fit. We also imposed to the object under study a conservative halo mass upper limit of $2 \times 10^{12} M_{\odot}$. The fits to the data are shown in Fig. 6, together with the BBS fits: for seven out of nine objects the NFW models are unacceptably worse than the BBS solutions. Moreover, in all objects, the CDM virial mass is too high: $M_{v i r} \sim 2 \times 10^{12} M_{\odot}$ and the resulting disk mass-to-light ratio too low ( $\lesssim 10^{-1}$ in the $I$-band).

\section{The Intriguing Evidence from Dark Matter Halos}

The dark halos around spirals emerge as an one-parameter family; the order parameter (either the central density or the core radius) correlates with the luminous mass. However, we do not know how it is related to the global structural properties of the dark halo, like the virial radius or the virial mass, unless we extrapolate out over the BBS profile. That is because the halo $\mathrm{RC}$, out to the outermost data, is completely determined by physical parameters, the central core density and the core radius, which have little counterpart in the gravitational instability/hierarchical clustering picture.

Caveat the above extrapolation, the location of spiral galaxies in the virtual space of virial mass/halo "central" density/stellar mass, that, on theorethical basis, should be roughly 3 -D random determined by several different and nonlinear physical processes, is remarkably found to degenerate and to lie on a 


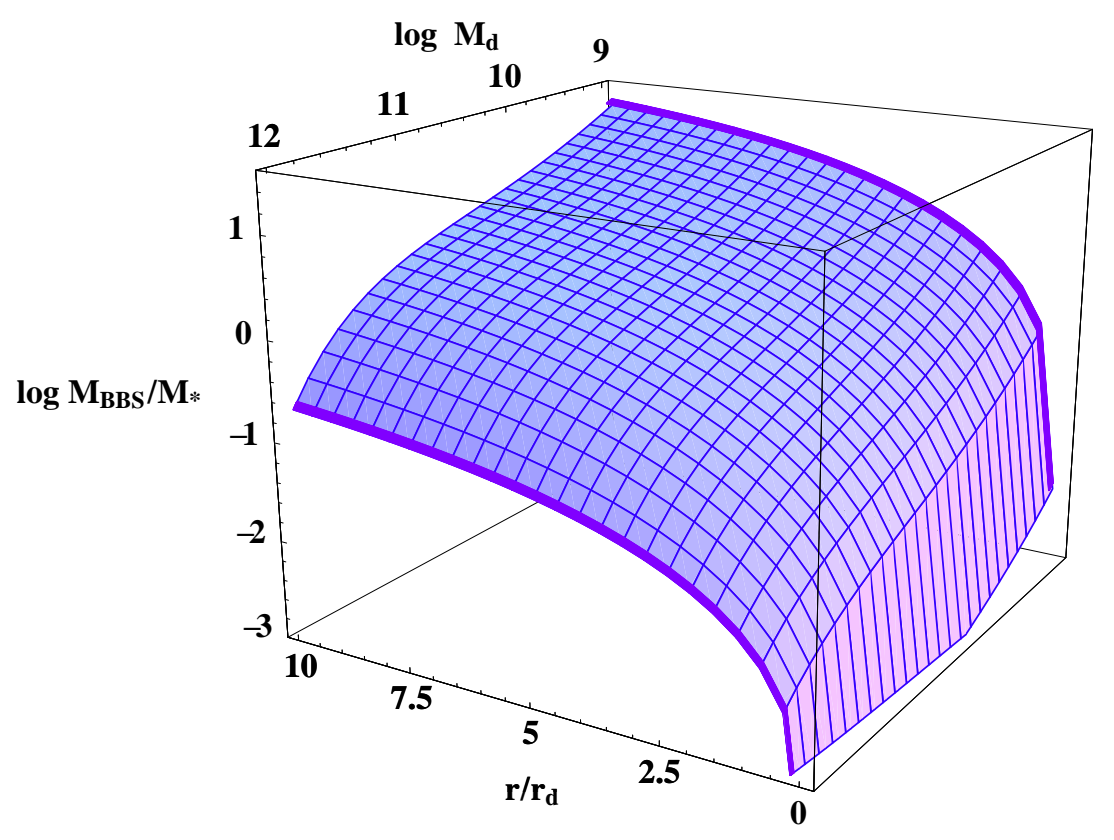

Fig. 7. The dark-to-luminous mass ratio as function of the normalized radius and the total disk mass.

curve. Indeed, in Fig.7 we show the dark-to-luminous mass ratio as function of the normalized radius and the total disk mass. The surface has been obtained by adopting the correlations between the halo and the disk parameters we found in our previous works (see Fig.3 [19]):

$$
\begin{aligned}
& \log r_{0}=9.10+0.28 \log \rho_{0}-3.49 \times 10^{10} \rho_{0}^{0.43} \\
& \log \rho_{0}=-23.0-0.077 \log M_{d}-9.98 \times 10^{-6} M_{d}^{0.43}
\end{aligned}
$$

and:

$$
\log r_{d}=4.96-1.17 \log M_{d}+0.070\left(\log M_{d}\right)^{2}
$$

from data in [16]. The dark-to-luminous mass ratio at fixed ratio increases as the total disk mass decreases; for example at $r=10 r_{d}$ it raises from $20 \%$ for massive disks $\left(M_{d}=10^{12} M_{\odot}\right)$ to $220 \%$ for smaller disks $\left(M_{d}=10^{9} M_{\odot}\right)$.

Two conclusive statements can be drawn: dark matter halos have an inner constant-density region, whose size exceeds the stellar disk length-scale. Second, there is no evidence that dark halos converge, at large radii, to a $\rho \sim r^{-2}$ (or steeper) profile.

The existence of a region of "constant" density $\rho_{0} \simeq \frac{\pi}{24} \frac{M_{\mathrm{vir}}}{r_{0}^{3}}$ and size $r_{0}$ is hardly explained within current theories of galaxy formation. Moreover, the 
evidence of a smooth halo profile is growing more an more in recent literature [e.g. 26, 27, 28, 29] and a number of different solutions have been proposed to solve this problem [e.g. 30, 31, 32, 33]. Let us stress, however, that we should incorporate all the intriguing halo properties described in this review.

\section{References}

1. A. Borriello, P. Salucci: MNRAS, 323, 285 (2001)

2. J.S. Bullock, T.S. Kolatt, Y. Sigad, R.S. Somerville, A.V. Kravtsov, A.A. Klypin, J.R. Primack, A. Dekel: MNRAS, 321, 559 (2001)

3. A. Burkert: ApJ, 447, L25 (1995)

4. A. Burkert, J. Silk: ApJ, 488, L55 (1997)

5. S. Cole, C. Lacey: MNRAS, 281, 716 (1997)

6. E. Corbelli, P. Salucci: MNRAS, 311, 411C (2000)

7. R. Flores, J.R. Primack: ApJ, 427, L1 (1994)

8. T. Fukushige, J. Makino: ApJ, 477, L9 (1997)

9. A.V. Kravtsov, A.A. Klypin, J.S. Bullock, J.R. Primack: ApJ, 502, 48 (1998)

10. S.S. McGaugh, W.J.G. de Block: ApJ, 499, 41 (1998)

11. B. Moore: Nature, 370, 629 (1994)

12. B. Moore, F. Governato, T. Quinn, J. Stadel, G. Lake: ApJ, 499, L5 (1998)

13. J.F. Navarro, C.S. Frenk, S.D.M. White: ApJ, 462, 563 (1996)

14. M. Persic, P. Salucci: MNRAS, 245, 577 (1990b)

15. M. Persic, P. Salucci: ApJS, 99, 501 (1995)

16. M. Persic, P. Salucci, F. Stel: MNRAS, 281, 27P (1996)

17. M.-H. Rhee: PhD thesis, Groningen University (1996)

18. P. Salucci, C. Ratnam, P. Monaco, L. Danese: MNRAS, 317, 488S (2000)

19. P. Salucci, A. Burkert: ApJ, 537L, 9S (2000)

20. P. Salucci, M. Persic: MNRAS, 309, 923 (1999)

21. J. Stil: Ph.D. Thesis, Leiden University (1999)

22. T.S. van Albada, J.S. Bahcall, K. Begeman, R. Sancisi: ApJ, 295, 305 (1985)

23. F.C. van den Bosch, B.E. Robertson, J. Dalcanton, W.J.G. de Blok: AJ , 119, 1579V (2000)

24. M. Persic, P. Salucci: in Dark and Visible Matter in Galaxies, ASP Conf. Ser., 117, ed. Persic \& Salucci (1997)

25. P. Salucci, A. Burkert: ApJ, 537, L9 (2000)

26. W.J.G. de Blok, S. S. McGaugh, A. Bosma, V. C. Rubin: ApJ, 552, 23 (2001)

27. C. M. Trott, R. L. Webs: astro-ph/0203196, in press (2002)

28. L. D. Matthews, J. S. Gallagher, III: astro-ph/0203188, in press (2002)

29. S. Blais-Ouellette, C. Carignan, P. Amram: astro-ph/0203146 to be published in: Galaxies: the Third Dimension, ASP Conf. Ser. (2002)

30. M. White, R.A.C.Croft: AJ, 539, 497 (2000)

31. P.J.E. Peebles: ApJ, 534, 127 (2000)

32. C. Firmani, E. D’Onghia, V. Avila-Reese, G. Chincarini, X. Hernandez: MNRAS, 315, 29 (2000)

33. A. El-Zant, I. Shlosman, Y. Hoffman: ApJ, 560, 636 (2001) 